\title{
Impact of the World Health Organization's Decision-Making Tool for Family Planning Clients and Providers on the quality of family planning services in Iran
}

\author{
Hamidreza Farrokh-Eslamlou, ${ }^{1}$ Siamak Aghlmand, ${ }^{2}$ Mohammad Eslami, $^{3}$ \\ Caroline S E Homer ${ }^{4}$
}

${ }^{1}$ Centre for Midwifery, Child and Family Health, Faculty of Health, University of Technology Sydney, New South Wales, Australia and Reproductive Health Research Center, School of Public Health, Urmia University of Medical Sciences, Urmia, Iran

${ }^{2}$ Reproductive Health Research Center, School of Public Health, Urmia University of Medical Sciences, Urmia, Iran

${ }^{3}$ Department of Population, Family and School Health, Ministry of Health and Medical Education, Tehran, Iran

${ }^{4}$ Centre for Midwifery, Child and Family Health, Faculty of Health, University of Technology Sydney, New South Wales, Australia

\section{Correspondence to}

Dr Caroline S E Homer, Centre for Midwifery, Child and Family Health, Faculty of Health,

University of Technology Sydney, PO Box 123, Broadway Sydney, New South Wales 2009, Australia;

caroline.homer@uts.edu.au

Received 2 January 2012 Revised 10 June 2013

Accepted 18 June 2013 Published Online First 14 August 2013

\begin{abstract}
Objective We investigated whether use of the World Health Organization's (WHO's) DecisionMaking Tool (DMT) for Family Planning Clients and Providers would improve the process and outcome quality indicators of family planning (FP) services in Iran.
\end{abstract}

Methods The DMT was adapted for the Iranian setting. The study evaluated 24 FP quality key indicators grouped into two main areas, namely process and outcome. The tool was implemented in 52 urban and rural public health facilities in four selected and representative provinces of Iran. A pre-post methodology was undertaken to examine whether use of the tool improved the quality of FP services and client satisfaction with the services. Quantitative data were collected through observations of counselling and exit interviews with clients using structured questionnaires.

Results Different numbers of FP clients were recruited during the baseline and the postintervention rounds ( $n=448$ vs 547 , respectively). The DMT improved many client-provider interaction indicators, including verbal and nonverbal communication $(p<0.05)$. The tool also impacted positively on the client's choice of contraceptive method, providers' technical competence, and quality of information provided to clients $(p<0.05)$. Use of the tool improved the clients' satisfaction with FP services (from $72 \%$ to $99 \% ; p<0.05)$.

Conclusions The adapted WHO's DMT has the potential to improve the quality of FP services.

\section{INTRODUCTION}

Aghlmand S, Eslami M, et al. I Fam Plann Reprod Health Care 2014;40:89-95.
In Iran, the prevalence of contraception is high, with almost $60 \%$ of women
Key message points

- The World Health Organization's Decision-Making Tool for Family Planning Clients and Providers, also known as the Family Planning Care Guideline (FPCG) flipchart, can be implemented in practice.

- This study has shown that the FPCG flipchart is a useful tool to improve the process and outcome quality indicators of family planning services.

- Further research is needed to assess the long-term behavioural impact of the WHO's Decision-Making Tool for Family Planning Clients and Providers.

reporting using modern contraceptive methods. ${ }^{1}$ This achievement is partly due to the easy availability of a wide range of modern contraceptives that are offered at community level services. Family planning $(\mathrm{FP})$ is also well integrated with other elements of reproductive health services. ${ }^{2}$ Despite these achievements in recent decades, there are still a number of challenges in relation to reproductive health in Iran. By the age of 19 years, $45 \%$ of women are either pregnant or have already given birth. Women in Iran experience high rates of unwanted pregnancy (up to $35 \%$ of pregnancies), ${ }^{3}$ illegal terminations of pregnancy ${ }^{4}$ and intermediate rates of maternal mortality. It is evident that there is still room for improvement in the provision of quality FP services. ${ }^{5}$ 
Research examining the quality and provision of FP services in existing health centres in Iran is scarce. Some studies have revealed that the quality of FP services is poor, especially in the terms of the protection of privacy and confidentiality and the provision of adequate information regarding contraceptive methods, ${ }^{6}{ }^{7}$ resulting in poor knowledge about methods and options among women. ${ }^{8}$ These studies also suggest that programmes to provide effective counselling and new ways to improve women's decision-making in relation to FP should be tested and evaluated.

There are many indicators that can be used to measure quality in FP services with different instruments being developed. Some are used to assess specific elements of quality while others assess quality in a comprehensive way. Donabedian ${ }^{9}$ has provided a generic framework for assessing the quality of health services in a way that examines three main dimensions of care: structure, process and outcome. This framework is a useful way of assessing FP services in Iran.

In addition, specifically in relation to FP, Bruce ${ }^{10}$ developed a framework for studying the quality of care in FP services that has six key indicators to assess the quality of services. The six indicators are: informed choice of methods, the provision of information to clients, technical competence of the provider, interpersonal relationship between client and provider, mechanisms to ensure continuity of care, and the appropriate provision of services. Appropriate provision of services refers to the placement of FP services so that they are convenient and acceptable to clients, and meet their needs. Services can be appropriately delivered through maternal and child health initiatives, that is, through postpartum services, comprehensive reproductive health services, adolescent services or employee health programmes. ${ }^{10}$

Further indicators of the quality of FP services outlined by the International Planned Parenthood Federation include rights-based indicators such as: women's right to information, access to services, informed choice, safe services, privacy and confidentiality, dignity, comfort, expression of opinion, continuity of care; as well as the facilitative supervision and training for providers and the effective provision of supplies, equipment and infrastructure. ${ }^{11}$ The quality of FP services includes access to information and counselling. Different types of counselling aids with standardised information have been designed and introduced in recent years to improve the quality of FP counselling. For example, the Population Council introduced the 'Balanced Counseling Strategy" ${ }^{\text {'2 }}$ and the World Health Organization (WHO) developed a 'Decision-Making Tool (DMT) for Family Planning Clients and Providers. ${ }^{13}$ The WHO DMT aims to improve the quality of FP services by improving the counselling process through better client-provider interactions, the provision of accurate information and by increasing informed choice. The DMT essentially is a generic, two-sided flipchart that providers use in their counselling discussions with FP clients.

There is limited evidence on the impact of the DMT on the quality of FP services. The DMT has been tested in several countries and been shown to improve the quality of counselling for FP clients, but current studies have only assessed the effects of the DMT on one or a few quality indicators of FP services such as acceptability, usability and health communication. ${ }^{13}$ In Mexico, clients reported that the DMT helped them understand the provider's explanations and made them feel more comfortable talking and asking questions during consultations. ${ }^{14}$ In Nicaragua, the DMT proved effective as a decision-making aid for clients, regardless of the client's level of education. ${ }^{15}$ Conversely, a follow-up study on long-term outcome of using DMT concluded that counselling alone, with or without DMT, did not influence contraceptive use rates. ${ }^{16}$

An important aim of the health system in Iran is to improve the quality of FP services. The DMT was therefore used in an effort to achieve this aim. The objective of the present study was to test whether training on and use of the DMT would improve the process and outcome quality indicators of FP services in Iran. We did not examine the structure of care or use structural indicators.

\section{METHODS}

An uncontrolled pre-post intervention study was undertaken to examine whether use of the WHO DMT improved the quality of FP services in 52 urban and rural public health facilities in the four Iranian provinces. Permission to carry out the study and ethical clearance were obtained from the ethical committee of the Urmia University of Medical Sciences.

\section{Pre-intervention stage}

The pre-intervention stage examined the quality of FP services before the DMT was introduced into practice. From July to September 2009, the pre-intervention stage of the study was conducted in the 52 urban and rural public health facilities in four provinces (West Azerbaijan, Semnan, Mazandaran, and Siatan and Baluchestan), purposively selected according to their different demographic, socio-economic, religious, cultural and ethnic backgrounds.

Sistan and Baluchistan (south-eastern) and West Azerbaijan (north-western) provinces have higher fertility and lower levels of socio-economic development than other provinces. Semnan and Mazandaran (located in central and northern Iran) have lower fertility and higher levels of socio-economic development in the country. There are also major ethnic variations among the selected provinces. Sistan and Baluchistan province is populated mainly by Baluchi and West Azerbaijan by two large ethnic groups namely Turks and Kurds, while the two other provinces contain 
mainly Persians. A considerable proportion of the population in both Sistan and Baluchistan and West Azerbaijan are Sunni Muslims, while the population in Semnan and Mazandaran are predominantly Shiites.

We selected one representative district from each province, namely Urmia, Semnan, Noor and Chabahar. Selecting these districts was based on the voluntary co-operation of health system managers and access of the researchers to these areas. One or two service providers, who had previously participated in FP counselling workshops, were selected from each facility, so that 78 service providers including 22 midwives, 31 family health skilled workers and 25 Behvarzes (primary health care providers in the rural settings) participated in the study. Clients were women who were presenting to the health services in order to receive information about new contraceptive methods or to change their current method.

Data were collected using two methods:

- Observation of the face-to-face counselling to evaluate the readiness of the facilities to deliver counselling, clientprovider interaction and selected clinical procedures,

- Exit interviews with clients as they left the facility.

Trained fieldworkers from each pilot district asked both the FP service providers and the clients for written informed consent to (1) to allow the counselling session to be evaluated by observation and (2) to be interviewed after the consultation.

The FP counselling sessions were evaluated by fieldworkers using an observation checklist to collect data on different aspects of the quality of counselling. A sample of 130 clients for each district was considered sufficient to demonstrate a $15 \%$ difference between the two pre-post intervention groups by using $80 \%$ power and $\alpha$ level of 0.05 . Each of 78 providers was observed with approximately six clients so a total of 448 consultations were observed during the baseline round. Exit interviews with clients were conducted in private, immediately following the same counselling sessions by fieldworkers using a structured questionnaire. This questionnaire collected socio-demographic data and the women's perception on the quality of the FP service and the counselling they had just received. Unique identifying information recorded on the observation and exit interview questionnaires for each client was used to link the data for each woman.

\section{Intervention stage}

The intervention was the use of the WHO's DMT also known as the Family Planning Care Guideline (FPCG) flipchart. All of the 78 service providers participated in a 2-day training workshop on the use of the FPCG flipchart. The workshop focused on how to use the FPCG flipchart and included technical information about topics that were new to the providers (such as Cyclofem ${ }^{\circledR}$, a monthly injectable combined contraceptive and dual protection, protecting from both sexually transmitted infections and unwanted pregnancy). Providers were then instructed to use the flipchart with every FP client for the next 6 months.

\section{Post-intervention stage}

The post-intervention phase took place at the end of the 6-month intervention phase. As in the preintervention stage, the same trained fieldworkers from each pilot district collected observational and interview data. Fieldworkers asked the FP service providers and the clients for written consent. The same service providers were observed but the clients participating were different. Some of the service providers (fewer than 10\%) had changed between the pre- and postintervention stage, but this was addressed with individual 2-day training. To ensure independence of the samples, clients were not admitted to the postintervention round of the study if they had been counselled in the baseline round. Clients were not interviewed in the post-intervention round of the study if they had been observed and interviewed in the baseline round.

\section{Measures and statistical analysis}

To evaluate the impact of the FPCG flipchart on the quality of FP services we compared the process and outcome indicators of FP services provided by the same providers in the periods before and after the intervention. The study employed the Quick Investigation of Quality (QIQ) ${ }^{17}$ assessment instrument that was previously used to evaluate the quality of the FP services. The QIQ was developed to monitor quality of care using observation of clientprovider interactions and exit interviews. The instrument uses $24 \mathrm{FP}$ care indicators to evaluate a range of dimensions affecting quality of care.

Eighteen of the 'quality of care' indicators were measured in both the observations and exit interviews. Some indicators were measured using only one method (e.g. delivering tailored information by provider was measured through observations of counselling). Two composite indicators on informed choice and decision-making were created from several questions (Table 1). Some questions (e.g. observing the principles of non-verbal communication) required a subjective judgment by the data collectors, however this subjectivity was consistent in the pre- and postintervention phases.

To simplify presentation of the data we have organised the indicators using the process and outcome paths of the Donabedian framework ${ }^{9}$ and the indicators of the service process were mapped to four of the six elements of the Bruce framework. Two other elements, namely 'mechanisms to ensure continuity of care' and 'the appropriate constellation of services', could not be evaluated by our study. ${ }^{10}$

We employed a binary scale (yes/no) to rate provider performance and client involvement on the 24 key 
Table 1 Questions aggregated to form indicators related to 'informed choice' and 'decision-making'

\begin{tabular}{|c|c|}
\hline Indicators & Questions \\
\hline \multirow[t]{3}{*}{$\begin{array}{l}\text { Informed choice of method by } \\
\text { client }\end{array}$} & $\begin{array}{l}\text { Did the provider provide information } \\
\text { about all available FP methods? }\end{array}$ \\
\hline & $\begin{array}{l}\text { Did the client select the FP method } \\
\text { by herself? }\end{array}$ \\
\hline & $\begin{array}{l}\text { Did the client select the FP method } \\
\text { after she knew about all available FP } \\
\text { methods? }\end{array}$ \\
\hline \multirow[t]{3}{*}{$\begin{array}{l}\text { Provider helped client with } \\
\text { decision-making process }\end{array}$} & $\begin{array}{l}\text { Did the provider help the client select } \\
\text { the FP method that was most } \\
\text { suitable for her situation? }\end{array}$ \\
\hline & $\begin{array}{l}\text { Did the consultation end with the } \\
\text { client's preferred method? }\end{array}$ \\
\hline & $\begin{array}{l}\text { Did the provider respond to all the } \\
\text { client's questions? }\end{array}$ \\
\hline
\end{tabular}

FP, family planning

behaviours or indicators measured through observations and interviews, although this is not the most suitable scale to measure all indicators such as 'satisfaction with the services'. These data were aggregated at the district and national level to yield proportions of consultations with positive outcomes for each variable.

Only participants with complete observation and interview data were included in the analysis $(n=448$ and $n=547$ in the pre-implementation and post-intervention stages, respectively). To ensure quality control of data collection, $10 \%$ of the counselling sessions were randomly selected and observed and rated by two investigators during both the pre- and post-intervention stages. To quantify the level of agreement between their ratings, kappa coefficient was calculated as 0.88 (95\% confidence interval 0.85-0.91). Descriptive statistics were used to analyse client characteristics. We used Student's $t$-test to test the significance of differences between baseline and post-intervention measures.

\section{RESULTS}

A total of 448 consultations during the baseline round and 547 consultations during the post-intervention round were observed. The proportion sampled from each district during the baseline and post-intervention rounds were: Urmia $21.0 \%$ vs $15.5 \%$, Chabahar $22.3 \%$ vs $25.3 \%$, Noor $33.7 \%$ vs $25.3 \%$ and Semnan $23.0 \%$ vs $33.8 \%$, respectively. In both baseline and post-intervention rounds most of the participants were 25-34 years of age, had a primary or high school education, had one or no children and no previous contraceptive method usage. There were no significant differences between clients' characteristics in the two rounds ( $p>0.05$ ).

The findings are presented according to the process and outcome indicators of FP services provided mostly by the same providers in the pre- and postintervention stages (Table 2).

\section{Process of the FP services}

To determine the impact of the tool on the process of FP services quality we used the four categories of indicators including contraceptive method selection; information given to clients; technical competence of the provider; and client-provider interpersonal interactions. These are presented below.

\section{Contraceptive method selection}

Compared with the pre-intervention phase, clients in the post-intervention stage participated more actively in selecting their contraceptive methods. Clients were more likely to be provided with opportunities to make informed choices in the post-intervention stage compared with pre-intervention. The proportions relating to informed choice of method by clients increased from $64 \%$ to $98 \%(p<0.01)$. There was also a significant increase post-intervention in the proportion of sessions in which providers assisted clients in the decision-making process from $53 \%$ to $96 \%(p<0.01)$.

Information given to the clients

Providers gave clients significantly more information on contraceptive methods in the post-intervention counselling sessions. For each of four indicators there was a significant increase in the post-intervention stage in the proportions of counselling sessions in which clients and providers met sufficient level of desired behaviours $(p<0.05)$. The improvements were in 'giving information about all FP methods' $(58 \%$ vs $80 \%$ ), 'giving information about efficacy of the chosen method' (54\% vs $87 \%$ ), 'giving information about using the chosen method' (81\% vs 98\%) and 'giving information about complications of the chosen method' (67\% vs 94\%).

Technical competence of the provider

The provider's technical competence, as reflected in three indicators, showed some improvement from preto post-intervention, although the difference was universally not statistically significant. The significant improvements were in 'delivering tailored information to the clients' ( $46 \%$ vs $90 \%, p<0.01$ ), 'correcting incorrect beliefs of the client about family planning' $(71 \%$ vs $86 \%, p=0.04)$ and 'answering all the client's questions' ( $89 \%$ vs $99 \%, p=0.04$ ).

\section{Client-provider interpersonal interactions}

For the indicators of 'observing the principles of verbal communication', 'observing the principles of non-verbal communication' and 'encouraging clients to speak' there was a significant increase at the post-intervention stage in the proportion of sessions in which clients met the minimum level of desired behaviour $(p<0.05)$. The FPCG flipchart did not have a statistically significant effect on many other aspects of client-provider interpersonal communication, for example, 'speaking with the client in a simple manner' $(p=0.89)$ and 'actively listening to the client' $(p=0.66)$. 
Table 2 Indicators to evaluate the impact of the Family Planning Care Guideline flipchart on the quality of family planning services

\begin{tabular}{|c|c|c|c|c|c|c|}
\hline & & \multirow[b]{2}{*}{$\begin{array}{l}\text { Baseline round } \\
(\%) \\
(n=448)\end{array}$} & \multirow[b]{2}{*}{$\begin{array}{l}\text { Post-intervention } \\
\text { round }(\%) \\
(n=547)\end{array}$} & \multirow[b]{2}{*}{$p^{*}$} & \multicolumn{2}{|c|}{ Data collection method } \\
\hline Indicators & cators & & & & Observation & $\begin{array}{l}\text { Exit } \\
\text { interview }\end{array}$ \\
\hline \multicolumn{7}{|c|}{ Process indicators } \\
\hline \multicolumn{7}{|c|}{ (a) Contraceptive method selection } \\
\hline 1 & Informed choice of method by client & 64 & 98 & $<0.01$ & $\checkmark$ & $\checkmark$ \\
\hline & Provider helped client with decision-making process & 53 & 96 & $<0.01$ & $\checkmark$ & $\checkmark$ \\
\hline \multicolumn{7}{|c|}{ (b) Information given to the clients } \\
\hline 3 & Giving information about all FP methods & 58 & 80 & 0.04 & $\checkmark$ & $\checkmark$ \\
\hline 4 & Giving information about efficacy of chosen method & 54 & 88 & $<0.01$ & $\checkmark$ & $\checkmark$ \\
\hline 5 & Giving information about using chosen method & 81 & 98 & 0.04 & $\checkmark$ & $\checkmark$ \\
\hline & Giving information about complications of chosen method & 67 & 94 & 0.03 & $\checkmark$ & $\checkmark$ \\
\hline \multicolumn{7}{|c|}{ (c) Technical competence of the provider } \\
\hline 7 & Delivering tailored information by provider & 46 & 90 & $<0.01$ & $\checkmark$ & \\
\hline 8 & Correcting wrong beliefs of the client about FP & 71 & 86 & 0.04 & $\checkmark$ & \\
\hline 9 & Answering all the client's questions & 89 & 99 & 0.04 & $\checkmark$ & $\checkmark$ \\
\hline \multicolumn{7}{|c|}{ (d) Client-provider interpersonal interactions } \\
\hline & Encouraging client to speak & 75 & 93 & 0.04 & $\checkmark$ & $\checkmark$ \\
\hline & Speaking with client in simple and understandable manner & 96 & 98 & 0.89 & $\checkmark$ & $\checkmark$ \\
\hline 12 & Possibility of client asking questions freely & 92 & 99 & 0.61 & $\checkmark$ & $\checkmark$ \\
\hline & Delivering simple explanations about chosen FP method & 95 & 100 & 0.72 & $\checkmark$ & $\checkmark$ \\
\hline 14 & Actively listening to the client & 93 & 99 & 0.66 & $\checkmark$ & $\checkmark$ \\
\hline 15 & Giving enough time to client to speak & 88 & 98 & 0.46 & $\checkmark$ & $\checkmark$ \\
\hline 16 & Empathy with the client & 46 & 57 & 0.28 & $\checkmark$ & $\checkmark$ \\
\hline 17 & Observing the principles of non-verbal communication & 85 & 98 & 0.04 & $\checkmark$ & \\
\hline 18 & $\begin{array}{l}\text { Observing the principles of verbal communication in } \\
\text { counselling }\end{array}$ & 75 & 97 & 0.04 & $\checkmark$ & \\
\hline 19 & Encountering privacy and confidentiality as client's right & 98 & 99 & 0.94 & $\checkmark$ & $\checkmark$ \\
\hline 20 & Greeting client by provider & 99 & 100 & 0.94 & $\checkmark$ & $\checkmark$ \\
\hline \multicolumn{7}{|c|}{ Outcome indicators } \\
\hline 21 & $\begin{array}{l}\text { Client chooses own contraceptive method by end of } \\
\text { session }\end{array}$ & 90 & 95 & 0.04 & $\checkmark$ & $\checkmark$ \\
\hline 22 & Obtaining overall good result from FP counselling session & 85 & 96 & 0.03 & $\checkmark$ & $\checkmark$ \\
\hline 23 & Client would recommend use of FP services to others & 56 & 98 & $<0.01$ & & $\checkmark$ \\
\hline & Client satisfaction with services & 72 & 99 & 0.03 & & $\checkmark$ \\
\hline
\end{tabular}

*Values for $p$ are calculated using Student's $t$-test.

FP, family planning.

\section{Outcome of the FP services}

Immediate effects on knowledge and attitude of FP clients using the FPCG flipchart were assessed using four outcome indicators. These were: ending the counselling session with a choice of contraceptive method; obtaining a good result from the FP counselling session; clients suggesting the FP service to others; and client satisfaction with services. There was a significant increase at post-intervention in the proportion of sessions in which counselling resulted in a choice of a contraceptive method $(p=0.04)$. The FPCG flipchart had a significant impact on clients' satisfaction with services. The overall satisfaction score rose from $72 \%$ to $99 \%(p=0.03)$. After the intervention, clients were more likely to recommend the FP service to others (from 55\% to 98\%; $p<0.01$ ).

\section{DISCUSSION}

We investigated whether use of the FPCG flipchart by health care providers would improve the quality of FP services in a number of clinics in one area in Iran. This study showed that the FPCG flipchart improved the quality of FP services despite limited training being provided to the providers.

The FPCG flipchart also improved the processes of FP services. This is significant as FP processes are often not ideal and issues such as supporting effective decision-making, providing usable information to the clients, and client-provider interpersonal interactions have been highlighted in other studies as being lacking at times. ${ }^{18}$ We showed that the FPCG flipchart increased the client's active involvement in the FP discussions, which is essential to decision-making. 
Our study, in common with others conducted in countries such as Mexico and Nicaragua, revealed improvements in the quantity and quality of information given to clients when the FPCG flipchart was used. ${ }^{14} 15$ There were some concerns that the flipchart's two-sided design would create some barriers between providers and clients, breaking eye contact and undermining the interaction. ${ }^{14}$ However, observation of counselling sessions showed that the principles of verbal and non-verbal communications were improved when the flip-chart was used.

Client-provider interaction is an essential component of the FP consultation. The 'interpersonal relations' element in the Bruce framework is essential in the client-provider transaction. ${ }^{10}$ It is this dimension that may strongly influence clients' satisfaction with the services, the probability of a return visit, increased compliance with the FP regimens, and better health outcomes. ${ }^{19}$ In such an interaction, the FP clients may be better able to make an informed selection and to use their chosen method correctly and consistently. Continued use of contraception would likely result in fewer instances of unwanted pregnancy and, potentially, a lower incidence of unsafe abortion. ${ }^{20}$

The FPCG flipchart used in the WHO DMT improved some client-provider interaction indicators including verbal and non-verbal communication, encouraging clients to speak, however it did not have a statistically significant effect on many other aspects of provider performance. There are two possible explanations for this lack of impact. First, some indicators are basic counselling and interpersonal communication skills and so did not relate directly to the content of the FPCG flipchart. Second, preintervention levels of provider performance were so high for some indicators that there was little room for improvement, such as 'speaking with the client in a simple and understandable manner' and 'encountering privacy and confidentiality'. The influence of the WHO DMT on client-provider interaction has been noted in other studies. In the study undertaken in Mexico, the clients reported that the WHO DMT made them feel more comfortable talking and asking questions during the consultation. ${ }^{14}$ In the Nicaraguan study, FP clients did become more forthcoming about their situation and their wishes when the WHO DMT was used. ${ }^{15}$

After 6 months using the FPCG flipchart, analysis of the counselling sessions and women's perceptions of the quality of FP services by the investigators confirmed that most counselling sessions ended with high levels of client satisfaction. Clients who chose FP methods after counselling with the FPCG flipchart were more likely to recommend this to others. Integration of the FPCG flipchart improved the locus of decision-making for clients. These improvements also occurred in both the Nicaraguan and Mexican studies, which reported marked shifts toward the client in the locus of decision-making after introduction of the DMT process. ${ }^{14} 15$

While the findings of this study are encouraging, there are a number of limitations to consider. First, observer bias is a source of potential error. Observation could have disrupted the privacy of the counselling and influenced providers' performance and clients' behaviour. However, observation has been used in many studies to evaluate the quality of health care and enables data on verbal and non-verbal communication to be collected. In support of this approach, a review of studies in this area has showed a high level of comparability in the results obtained from observation and client exit interviews on most quality of FP care indicators. ${ }^{17}$ In the present study, two data sources, namely observation and exit interview, were combined and it is likely that this would have reduced observer bias.

A second limitation is the need for assessment of the long-term behavioural impact of the DMT. The findings of our research demonstrated short-term outcomes such as satisfaction of the clients with FP services and immediate choices. The present study did not measure the impact of the FPCG flipchart on method continuation or any long-term impacts on unwanted pregnancy and unsafe abortion. This is necessary as a quasi-experimental study in Nicaragua revealed that using the WHO DMT had no effect on longer-term contraceptive continuation. ${ }^{16}$ Another randomised control trial demonstrated that structured counselling using DMT had little impact on continuation of contraceptive use. ${ }^{21}$

Measuring client satisfaction using only one dichotomous question is another limitation of this study. Clients' satisfaction with services has been linked to FP service quality, and perceptions of satisfaction reflects a client's attitudes about services not her beliefs. ${ }^{22}$ Therefore, clients may be satisfied with services even if they are of lower quality. Further studies should pay careful attention to designing a wide range of questions and approaches for evaluating client satisfaction.

Another limitation of the present study is the representativeness of the sample of clinics, providers and clients. Our clinics were rural and urban health centres in the government sector, which limits the generalisability to private clinics. Our study groups were only new FP clients and those considering changing FP methods, and these individuals may not behave like clients who just want to continue with their chosen method. Usually, the majority of FP clients are repeat users, although many may be experiencing problems with their current method. Some of the providers changed during the study, although the exact numbers were not recorded. The final limitation of the study was the lack of a simultaneous control group. We consider it unlikely, however, that other factors contributed to the observed changes in these clinics. 


\section{CONCLUSION}

In this study the adapted WHO DMT known as the FPCG flipchart improved decision-making, informed choice and client satisfaction with services. The DMT may have the potential to improve the quality of FP services in process and outcome paths of the quality of care.

Acknowledgements The study was conducted by the Reproductive Health Research Centre of Urmia University of Medical Sciences in collaboration with the Centre for Midwifery, Child and Family Health, Faculty of Health at the University of Technology Sydney (UTS) and the Family Planning Council of the Ministry of Health and Medical Education of Iran. The authors would like to thank the service providers and women who participated in this research in Urmia, Chabahar, Noor and Semnan districts of Iran.

Funding This study was funded by the United Nations Population Fund (UNFPA) office in Iran.

\section{Competing interests None.}

Ethics approval Ethical approval for the study was granted by the Research Ethical Committee of Urmia University of Medical Sciences in Iran.

Provenance and peer review Not commissioned; externally peer reviewed.

\section{REFERENCES}

1 World Health Organization Regional Office for the Eastern Mediterranean. Country profiles: Islamic Republic of Iran. http://www.emro.who.int/emrinfo/index.aspx?Ctry=ira [accessed 10 February 2009].

2 Anonymous. Iranian health houses open the door to primary care. Bull WHO 2008;86:585-586.

3 Abbasi-Shavazi J, Hosseini-Chavoshi M, Aghajanian A, et al. Unintended pregnancies in the Islamic Republic of Iran: levels and correlates. Asia Pac Popul J 2004;19:27-38.

4 Malekafzali H. Population control and reproductive health in the Islamic Republic of Iran. Arch Iran Med 2004;7:247-250.

5 Blanc AK, Curtis S, Croft T. Does Contraceptive Discontinuation Matter? Quality of Care and Fertility Consequences (MEASURE Evaluation Technical Report Series, No. 3). 1999. http://pdf.usaid.gov/pdf_docs/PNACJ177.pdf [accessed 10 February 2009].

6 Nakhaee N, Mirahmadizadeh AR. Iranian women's perceptions of family-planning services quality: a client-satisfaction survey. Eur J Contracept Reprod Health Care 2005;10:192-198.

7 Mohammad-Alizadeha S, Wahlströmb R, Vahidi R, et al. Women's perceptions of quality of family planning services in Tabriz, Iran. Reprod Health Matters 2009;17:171-180.
8 Simbar M, Ahmadi M, Ahmadi G, et al. Quality assessment of family planning services in urban health centers of Shahid Beheshti Medical Science University, 2004. Int J Health Care Qual Assur Inc Leadersh Health Serv 2006;19:430-442.

9 Donabedian A. Quality assurance: structure, process and outcome. Nurs Stand 1992;7(11 Suppl. QA):4-5.

10 Bruce J. Fundamental elements of the quality of care: a simple framework. Stud Fam Plann 1990;21:61-91.

11 Finger WR. Choice, access, information are among client's rights. Network 1993;14:24-26.

12 Martin A. Enhancing Quality for Clients: The Balanced Counseling Strategy. FRONTIERS Program Brief No. 3. Washington, DC: Population Council, 2003.

13 Johnson SL, Kim YM, Church K. Towards client-centered counseling: development and testing of the WHO DecisionMaking Tool. Patient Educ Couns 2010;81:355-361.

14 Kim YM, Kols A, Martin A, et al. Promoting informed choice: evaluating a decision-making tool for family planning clients and providers in Mexico. Int Fam Plan Perspec 2005;31:162-171.

15 Kim YM, Davila C, Tellez C, et al. Evaluation of the World Health Organization's family planning decision-making tool: improving health communication in Nicaragua. Patient Educ Couns 2007;66:235-242.

16 Chin-Quee DS, Janowitz B, Otterness C. Counseling tools alone do not improve method continuation: further evidence from the decision-making tool for family planning clients and providers in Nicaragua. Contraception 2007;76:377-382.

17 The MEASURE Evaluation Project. Quick Investigation of Quality (QIQ); A User's Guide for Monitoring Quality of Care in Family Planning. MEASURE Evaluation Manual Series, No. 2. Chapel Hill, NC, USA: Carolina Population Center, University of North Carolina, 2001.

18 Bessinger RE, Bertrand JT. Monitoring quality of care in family planning programs: a comparison of observations and client exit interviews. Int Fam Plan Perspect 2001;27:63-68.

19 León FR, Monge R, Zumarán A, et al. Length of counseling sessions and the amount of relevant information exchanged: a study in Peruvian clinics. Int Fam Plan Perspect 2001;27:28-33.

20 Roter D, Hall J. Doctors Talking with Patients. Patients Talking with Doctors: Improving Communication in Medical Visits. Westport, CT: Auburn House, 1992.

21 Smith R, Ashford L, Gribble J, et al. Family Planning Saves Lives. Washington, DC: Population Reference Bureau, 2009.

22 Langston AM, Rosario L, Westhoff CL. Structured contraceptive counseling - a randomized controlled trial. Patient Educ Couns 2010;81:362-377.

23 Linder-Pelz SU. Toward a theory of patient satisfaction. Soc Sci Med 1982;16:577-582. 